\title{
Impacto de la erosión hídrica sobre la rentabilidad de los productores agrícolas en el partido de Tres Arroyos, Región Pampeana Austral, Argentina
}

\author{
Impact of water erosion on the profitability of agricultural \\ producers in the partido of Tres Arroyos, Pampean Austral \\ Region, Argentina
}

\author{
Nahuel David Sequeira ${ }^{1}$ \\ Universidad Nacional del Centro de la Provincia de \\ Buenos Aires-CONICET, Argentina \\ Patricia Vazquez ${ }^{2}$ \\ Universidad Nacional del Centro de la Provincia de \\ Buenos Aires-CONICET, Argentina
}

\begin{abstract}
Resumen
Mundialmente, la erosión hídrica se constituye como uno de los procesos de degradación de suelos más relevantes. Los objetivos del presente trabajo son: a) estimar la tasa de erosión hídrica actual y potencial y la variación de la pérdida total de suelos agrícolas del partido de Tres Arroyos (Región Pampeana Austral), para las campañas agroproductivas 1989/90, 2002/03 y 2019/20 y b) desarrollar un acercamiento a la estimación de la pérdida económica a causa de la merma de la productividad
\end{abstract}

1 Doctorando del programa de Ciencias Agrarias de la Universidad Nacional de Rosario (Santa Fe, Argentina). Lic. en Diagnóstico y Gestión Ambiental. Becario doctoral del Consejo Nacional de Investigaciones Científicas y Técnicas (CONICET). Centro de Estudios Sociales de América Latina, Facultad de Ciencias Humanas, Universidad Nacional del Centro de la Provincia de Buenos Aires-CONICET. Paraje Arroyo Seco s/n, Tandil, Buenos Aires, Argentina. Correo electrónico: nahuelsequeira@conicet.gov.ar. (iD https:// orcid.org/0000-0003-2206-4411

2 Doctora en Ciencias Agrarias. Investigadora Independiente del CONICET. Centro de Estudios Sociales de América Latina, Facultad de Ciencias Humanas, Universidad Nacional del Centro de la Provincia de Buenos Aires-CONICET. Paraje Arroyo Seco s/n, Tandil, Buenos Aires, Argentina. Correo electrónico: patriciavazquez@conicet.gov.ar. iD https://orcid.org/0000-0002-4209-4901 
del suelo originada por la erosión hídrica en el área de estudio. Metodológicamente, se calcularon las tasas de erosión y pérdida de suelo y su evolución temporal, y se determinó la disminución del rendimiento de tres cultivos principales y su impacto económico. El partido demuestra, desde 2002/03, un aumento en la pérdida productiva, debido al incremento del área sembrada y la existencia de procesos erosivos; traduciéndose en un perjuicio económico sobre los productores, y en la necesidad de desarrollar estrategias enfocadas hacia un modelo de producción más sustentable.

Palabras clave: avance agrícola; procesos erosivos; disminución de ganancias; impactos ambientales; agricultura sustentable.

\begin{abstract}
Worldwide, water erosion is one of the most relevant soil degradation processes. The objectives of the present work are: a) to estimate the current and potential water erosion rate, and the variation of the total loss of agricultural soils in the partido (roughly translated as district) of Tres Arroyos (Pampean Austral Region) in the province of Buenos Aires, Argentina, for the 1989/90, 2002/03, and 2019/20 agro-productive campaigns; and b) to develop an approach to estimating the economic loss due to the decrease in soil productivity caused by water erosion in the study area. Methodologically, the rate of erosion and rate of soil loss and their temporal evolution were calculated, and the decrease in yield of three main crops and their economic impact was determined. Since 2002/03, the partido (district) shows a decrease in productivity, due to the increase in the area of cultivated land and the existence of erosive processes, translating into economic damage to producers, and highlighting the urgency to develop strategies focused on a more sustainable production model.
\end{abstract}

Keywords: Agricultural advance; Erosive processes; Decrease in profits; Environmental impacts; Sustainable agriculture.

\title{
Introducción
}

Dentro de los recursos naturales, el suelo ejerce un rol relevante, debido a sus múltiples funciones ecológicas y a aquellas asociadas a satisfacer las necesidades de la sociedad. A pesar de ello, es considerado no renovable en la escala humana, desarrollándose lentamente y demostrando una notable variabilidad espacial (Díaz, Anaya \& de la Rosa, 2011).

La utilización de este recurso por parte de los seres humanos, ha sido ininterrumpida desde la invención de las técnicas agrícolas, a través de distintos procesos (Leal \& Lozano, 2012). Entre estos, aquellos que generan una depreciación de la capacidad productiva de los suelos son denominados degradativos, destacándose a la erosión (hídrica y eólica) como uno de los más impactantes (FAO, 1990; Bouchoms, Wang, Vanacker, \& Oost, 2019).

En términos globales, la erosión puede establecerse como un fenómeno natural vinculado particularmente a factores geológicos. Sin embargo, diversas acciones antrópicas, como el uso intensivo de las tierras 
agrícolas, el cambio de coberturas y usos del suelo y manejos inadecuados, suelen acelerar e intensificar el proceso (FAO, 1990; Toy, Foster, \& Renard, 2002; Cristeche, 2009; Gvozdenovich, Pérez, Novelli, \& Barbagelata, 2017; Concepción, Lizaga, Gaspar, Quijano, \& Navas, 2018).

Con relación a ello, en la literatura existente es plausible observar intentos de separación entre erosión natural y erosión antrópica; relacionándose el primer concepto con un papel preponderante de los factores naturales, y el segundo con el predominio de otros de carácter socioeconómico. A pesar de esto, es necesario considerar que todos estos factores inciden en distinta medida en el grado y mantenimiento de la cobertura vegetal del suelo, cuya remoción suele considerarse la causa principal de la erosión, por lo que aparecen grandes dificultades al momento de distinguir entre los dos tipos mencionados (Cristeche, 2009).

Mundialmente, la erosión hídrica se presenta como uno de los fenómenos más significativos de degradación del suelo, desencadenando graves impactos ecológicos y elevados costos económicos. Así, mediante sus efectos sobre la producción agrícola, la infraestructura y la calidad del agua que, al mismo tiempo, perjudica la calidad de vida de la población, este proceso amenaza la seguridad alimentaria, constituyéndose como una barrera al momento de alcanzar el desarrollo sustentable (Nájera, Bojórquez, Flores, Murray \& González, 2016; Sartori et al., 2019).

Este panorama, en el cual nos enfrentamos a un incremento de la pérdida de suelos por procesos erosivos, se revela como uno de los problemas ambientales esenciales de los territorios en los que se desarrolla la agricultura (Cristeche, 2009); ya que, alrededor de una cuarta parte de las tierras cultivables del planeta, manifiestan signos de degradación y/o erosión (Ferreras, Toresani, Faggioli, \& Galarza, 2015). Por lo tanto, según García, Ruiz, Savé y Herrera (2013) y Nearing, Xie, Liu y Ye (2017), las prácticas agrícolas son una de las principales responsables de la erosión del suelo.

Adicionalmente, para muchos países de Latinoamérica, esta problemática se convirtió en una de las que ocasionan impactos negativos más graves, favoreciendo el origen de estados más pobres y con mayor cantidad de riesgos ambientales (Gvozdenovich et al., 2017).

Con relación a lo mencionado, se afirma que la erosión afecta tanto a países desarrollados o en desarrollo, aunque la situación en estos últimos resulta particularmente preocupante, dado que sus economías suelen 
sustentarse primordialmente en la agricultura y la explotación de recursos naturales; por lo que ven amenazada su base productiva (Cristeche, 2009).

Dentro de este contexto, la Argentina, a pesar de haber sobresalido históricamente por sus ventajas comparativas para el desarrollo agropecuario, alcanzando el sector un desempeño económico sin precedentes en los últimos años, no se encuentra exenta de dicha problemática (Cristeche, 2009). En los últimos 60 años, en el país creció significativamente la superficie afectada por erosión hídrica, pasando de 18 millones de ha en 1956, a más de 64 millones en 2015 (Gvozdenovich et al., 2017); y, al igual que en el resto del mundo, la mencionada situación se manifiesta como uno de los principales impactos negativos de las regiones dedicadas a la explotación agrícola, tal es el caso de la Región Pampeana Argentina (RPArg).

Con la finalidad de intentar remediar este problema, en el país comenzó a adoptarse a fines de la década de 1980 la Siembra Directa (SD), sobresaliendo como una de las estrategias productivas más divulgadas para enfrentar a la erosión y llegando a aplicarse en más del 75\% de la superficie agrícola (López, Duval, Martínez \& Galantini, 2015). La SD es una técnica basada en el cultivo de la tierra sin arado previo, por lo que no se remueven los rastrojos de los cultivos anteriores y se asegura una cobertura permanente del suelo, mejorando sus condiciones físicas, químicas y biológicas (Castilla, 2013); constituyendo una alternativa para reducir el escurrimiento y la erosión en las tierras cultivables (Armand, Bockstaller, Auzet \& Van Dijk, 2009).

Pese a ello, Casas (2015) afirma que aproximadamente 64,6 millones de hectáreas del territorio argentino resultaban afectadas por erosión hídrica, siendo este valor un $150 \%$ más que las 25 millones calculadas por FECIC-PROSA (1988) en estudios anteriores.

Teniendo presente lo hasta aquí dicho, es probable aseverar que la Región Pampeana Austral (RPA), inserta dentro de la RPArg, también refleja la citada coyuntura. En este sentido, Cerdá, Sarandón y Flores (2014) sugieren que, durante mucho tiempo, el modelo de producción se encontró sustentado en una alternancia entre la actividad ganadera y la agricultura. Pese a ello, una hipotética mayor rentabilidad de los cultivos, en asociación con la falta de comprensión acerca de los servicios ambientales generados por la ganadería pastoril, instigó una apreciable agriculturización en la región. Por esta razón, comenzó a revelarse una rauda transformación 
del paisaje, originándose una serie de hondas modificaciones en los suelos de los agroecosistemas; destacándose a la erosión y sus subsecuentes pérdidas económicas como algunas de las repercusiones más relevantes.

Incluso cuando es altamente conocido que los impactos negativos de la erosión revisten gravedad, y existe un enorme número de investigaciones que abordan sus efectos en relación al proceso de agriculturización a nivel de región o cuenca; permanecen siendo escasas aquellas que lo hacen a niveles inferiores, tal es el caso de la escala de partido.

Tomando lo hasta aquí descripto y considerando que dentro de los efectos más analizados del proceso de erosión se encuentra el impacto sobre la actividad agrícola, en relación a la reducción de la productividad y su repercusión sobre la rentabilidad del agricultor (Colombo, 2004); los objetivos del presente trabajo son: a) estimar la tasa de erosión hídrica actual (A) y potencial (EP) y la variación de la pérdida total de suelos agrícolas del partido de Tres Arroyos (en la RPA), para las campañas agroproductivas 1989/90, 2002/03 y 2019/20 y b) desarrollar un acercamiento a la estimación de la pérdida económica a causa de la merma de la productividad del suelo originada por la erosión hídrica en el área de estudio.

\section{Área de estudio}

El partido de Tres Arroyos se ubica en el sudeste de la provincia de Buenos Aires (Figura 1), posee una superficie de 596288 ha, limita hacia el este con el partido de San Cayetano, hacia el norte con los partidos de Adolfo Gonzáles Chaves y Coronel Pringles, hacia el oeste con el partido de Coronel Dorrego y hacia el sur con el océano Atlántico (Mikkelsen, 2013); y, además, posee una altitud media de $100 \mathrm{msnm}$ y una población de 57110 habitantes. 
Figura 1. Ubicación del área de estudio

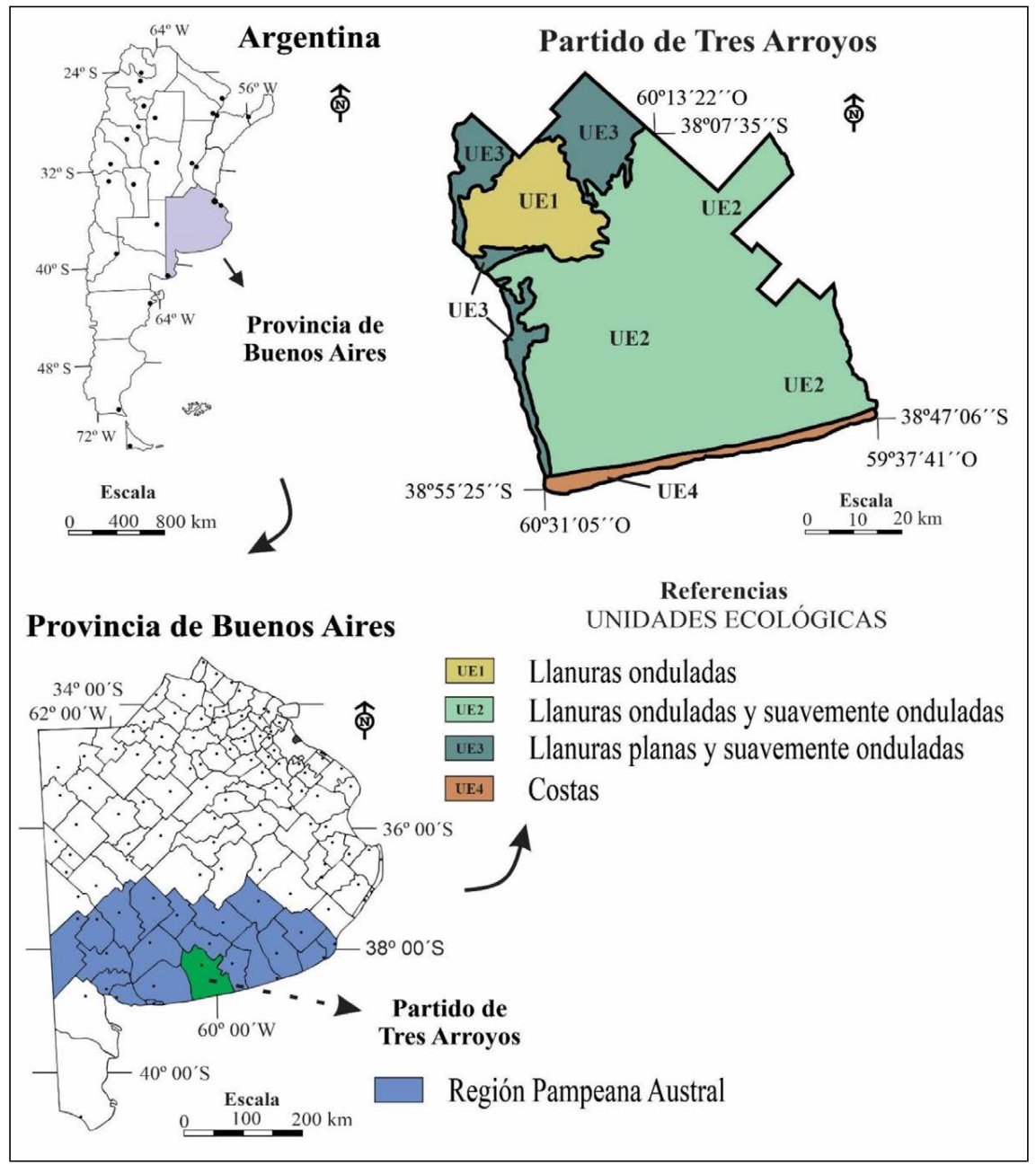

Fuente: Elaboración personal en base a Vazquez, Zulaica, Mickelsen \& Sacido (2018).

Históricamente, el partido es reconocido como un polo agrícola, aunque también se destaca el desarrollo de un importante nivel de industria en la zona urbana y peri-urbana; especialmente de la metalmecánica y la alimenticia, estando la primera ligada a la generación de infraestructura rural (silos, galpones) y la venta de maquinaria agropecuaria, y la segunda asociada con la transformación de materias primas. Igualmente, considerando que existe un alto porcentaje de superficie con suelos con aptitud agrícola, 
la producción agropecuaria es la principal fuente de actividad e ingresos (Carbone y Piccolo, 2002; Villanueva, Cazenave, \& Bilello, 2009).

Según Vázquez et al. (2018), quienes efectuaron una zonificación ecológica del área, mediante la integración de atributos bióticos y abióticos (geomorfológicos, edáficos, drenaje y vegetación) en el entorno de un sistema de información geográfica, puede subdividirse en cuatro unidades ecológicas (UE). Estas, denominadas UE1 (Llanuras onduladas), UE2 (Llanuras onduladas y suavemente onduladas), UE3 (Llanuras planas y suavemente onduladas) y UE4 (Costas) presentan características particulares, tal como demuestra la Tabla 1. Asimismo, el trabajo destaca que entre los años 2002 y 2017, se produjo un aumento del $49,11 \%$ de la superficie agrícola y una disminución del 35,56\% del área ganadera.

\section{Tabla 1. Principales características de las Unidades ecológicas que componen al partido}

\begin{tabular}{|c|l|}
\hline UE1* & $\begin{array}{l}\text { Ocupa un } 10 \% \text { de la superficie total del partido. Presenta paisajes de relieves ondulados, } \\
\text { con pendientes de entre el } 3 \text { y } 5 \% \text {. Imperan suelos clasificados a nivel de subgrupo como } \\
\text { Argiudoles típicos (Series Laprida y Necochea), cuya susceptibilidad a la erosión confor- } \\
\text { ma un factor limitante. }\end{array}$ \\
\hline UE2* & $\begin{array}{l}\text { Abarca un } 70 \% \text { de la superficie y está conformada por paisajes de relieves ondulados y sua- } \\
\text { vemente ondulados con pendientes de entre el } 1 \text { y 3\%. Imperan los suelos clasificados a nivel } \\
\text { de subgrupo como Argiudoles típicos (Series Laprida y Necochea) y Argiudoles petrocálcicos } \\
\text { (Serie Tres Arroyos), aunque pueden asociar Hapludoles típicos (Serie Ochandio) en pendien- } \\
\text { tes más pronunciadas y Hapludoles tapto nátricos (Serie La Pandorga) y Natracuoles típicos } \\
\text { (Serie Mechongué) en áreas con drenaje deficiente y en las proximidades de cursos de agua. }\end{array}$ \\
\hline UE3 & $\begin{array}{l}\text { Ocupa un } 15 \% \text { del partido y el promedio de las pendientes es inferior al 1 \%. Se caracteriza } \\
\text { por la presencia de un drenaje superficial e interno imperfecto y asocia vías de escurrimiento. } \\
\text { Los suelos predominantes son Argiudoles ácuicos (Serie Lobería). No obstante, en las lomas } \\
\text { aparecen Argiudoles tipicos (Series Laprida y Necochea) y en áreas con drenaje deficiente } \\
\text { Natracuoles típicos (Serie Mechongué). Como consecuencia de las limitaciones asociadas } \\
\text { con el drenaje deficiente, algunos sectores de este paisaje conservan pastizales nativos, ya } \\
\text { que los suelos anegables impiden o dificultan el desarrollo de actividades productivas. En } \\
\text { consecuencia, las actividades predominantes en este paisaje son las ganaderas. }\end{array}$ \\
\hline UE4 & $\begin{array}{l}\text { Ocupa un } 5 \% \text { de la superficie, presentando en general geoformas de acumulación (du- } \\
\text { nas) y playas. El desarrollo de los suelos es muy débil y, cuando existe, es incipiente. Los } \\
\text { suelos dominantes integran el subgrupo Cuarcipsamentes típicos (Serie Paraje Arenas } \\
\text { Verdes), cuya textura superficial y subsuperficial (areno-franca) favorecen un drenaje } \\
\text { excesivo. En menor medida se presentan Hapludoles énticos (Serie Estancia Santa Ana) y } \\
\text { Hapludol fluvéntico (Serie Paraje Los Ángeles). Este paisaje no es apto para el desarrollo } \\
\text { de actividades agropecuarias. }\end{array}$ \\
\hline
\end{tabular}

*Contienen suelos profundos y, debido a su aptitud agroecológica, los pastizales han sido sustituidos por cultivos, siendo esta última la actividad predominante.

Fuente: Vazquez et al. (2018) y Vazquez, Ruiz, Zulaica, Sequeira \& Daga (2019). 


\section{Marco metodológico}

La elección del área de estudio se fundamentó en que el partido de Tres Arroyos (inserto en la RPA), representa un notable ejemplo del proceso de agriculturización, el cual se manifiesta de forma diferenciada hacia el interior de las UE que lo conforman (Vazquez et al., 2018). De igual forma, en este trabajo solo fueron analizadas aquellas en las que se desarrollan las actividades agropecuarias (UE1, UE2 y UE3).

En cuanto a las fechas escogidas, se destaca que es a partir de la campaña agroproductiva 1989/90 cuando comienza a iniciarse un importante desarrollo de la actividad agrícola en la región; en 2002/03 el avance de la agricultura cobra aún más relevancia debido a la sustancial adopción de la SD por parte de los agricultores (Vazquez, 2004); y, por último, la campaña 2019/20 es aquella que se corresponde con las bases de datos oficiales más actualizadas.

En pos de lograr un mejor nivel organizacional, se decidió dividir este apartado en dos etapas. Inicialmente, se procedió a efectuar el cálculo acerca A y EP y su variación temporal. A continuación, se llevó a cabo el análisis sobre la pérdida económica de los productores agrícolas, debida a la disminución de la productividad del suelo como consecuencia del proceso de erosión hídrica.

\section{Primera etapa}

En primer lugar, como ya fue mencionado, se consideró el trabajo en el que se analizaron las diversas UE que conforman al partido de Tres Arroyos. A partir de esta información, en conjunto con la aportada por el Atlas de suelos de la República Argentina (Instituto Nacional de Tecnología Agropecuaria (INTA), 1990), fue posible determinar las series de suelo predominantes en cada una de las mencionadas unidades; las cuales se constituyen como insumos fundamentales al momento de estimar los valores de A y EP.

La valoración de las tasas de erosión fue concretada mediante la utilización de la Ecuación Universal de Predicción de Erosión Hídrica (USLE en inglés; Ecuación 1) definida por Wischmeier y Smith (1978). Esta herramienta ha sido utilizada de manera considerable por distintos investigadores (Nájera et al., 2016), ya que posee una enorme capacidad de aplicación a distintas áreas, bajo costo cuando existen datos de base 
disponibles y gran operatividad para identificar regiones susceptibles ante el proceso de erosión (Hernández \& Dufilho, 2012).

$$
A=E * K^{*} L * S * P
$$

Donde: A: Tasa de erosión actual $\left(\mathrm{t} \mathrm{ha}^{-1} \mathrm{año}^{-1}\right), \mathrm{R}$ : Erosividad de las lluvias ( $\left.\mathrm{hJ} \mathrm{cm} \mathrm{m}^{-2} \mathrm{~h}^{-1}\right), \mathrm{K}$ : Susceptibilidad del suelo a la erosión hídrica $(\mathrm{t}$ $\left.\mathrm{m}^{2} \mathrm{~h} \mathrm{ha}^{-1} \mathrm{hJ}^{-1} \mathrm{~cm}^{-1}\right)$, L: Longitud de la pendiente (m), S: Pendiente (\%), C: Factor cultivo o cobertura (adimensional) y P: Factor práctica conservacionista (adimensional).

El factor R alude a la erosividad de la lluvia y simboliza los factores de lluvia y escurrimiento (Gaitán et al., 2017). Para su obtención, fue preciso tener en cuenta los dominios edáficos que caracterizan al área en estudio, los cuales se encuentran analizados y publicados por Irurtia y Cruzate (2002).

Con respecto al factor $\mathrm{K}$, remite a la susceptibilidad del suelo frente a la erosión o erodabilidad, y para su estimación se demanda poseer información edáfica específica de los perfiles de suelos, tal como el porcentaje de materia orgánica, limo y arena muy fina, el grado de estructura, la permeabilidad y el porcentaje de arcilla (Gaitán et al., 2017).

En referencia a los factores L (largo de la pendiente) y S (gradiente de la pendiente), son estudiados de forma conjunta y se los designa como factor topográfico (LS), el cual define el aporte que efectúa el relieve a la erosión hídrica. La información fue obtenida de datos provenientes de Irurtia y Cruzate (2002) y la consulta en línea de las cartas de suelos (escala 1:50000) del INTA, respectivamente.

Por último, tanto el factor $\mathrm{C}$ como el $\mathrm{P}$ se encuentran sujetos de manera directa al tipo de manejo que los distintos productores realizan en sus establecimientos (Gaitán et al., 2017).

El producto resultante de estos seis factores mencionados permite estimar los valores de A para una situación específica de suelo, relieve, clima, cultivo y manejo. Asimismo, la valoración de la EP se logra mediante el producto de los factores R, K y LS y es definida como la máxima posible para un sitio determinado, es decir, considerando un suelo desnudo durante la totalidad del año. 
Con el fin de llegar a efectuar los cálculos necesarios para el desarrollo del objetivo, se empleó el software desarrollado por Gvozdenovich, Barbagelata y López (2015) que facilita, a través de la aplicación de la ecuación mencionada anteriormente, llevar a cabo los cómputos de pérdidas de suelo según la información disponible para cada caso.

Finalmente, la información adquirida acerca de A y EP en conjunto con la concerniente a la superficie destinada a los principales cultivos del área, se utilizó en la determinación de la pérdida total de suelos $\left(\mathrm{t}\right.$ año $\left.{ }^{-1}\right)$ y su modificación entre las campañas analizadas. Para ello, se seleccionaron las series más representativas de las tres UE y se calcularon valores promedio de su A y EP, los que más tarde se multiplicaron por la superficie agrícola previamente mencionada para las campañas 1989/90, 2002/03 y 2019/20, alcanzándose los valores de pérdida total de suelos actual (PTSA) y pérdida total de suelos potencial (PTSP).

\section{Segunda etapa}

Para concretar el análisis acerca de la pérdida económica fue necesario, por un lado, establecer la disminución real del rendimiento de los cultivos por unidad de pérdida de suelo, para poder estimar la pérdida de producción total; y por otro, llevar a cabo una ponderación entre esta pérdida y el precio de los granos en el mercado. Inicialmente, el impacto de la erosión hídrica sobre el rendimiento de los cultivos se estimó a partir de la utilización de la Ecuación 2, adaptada de Gaitán et al. (2017)

$$
\Delta y_{\mathrm{i}}=(\mathrm{a} * \mathrm{~b})
$$

Donde: $\Delta \mathrm{y}_{\mathrm{i}}$ : disminución real del rendimiento $\left(\mathrm{kg} \mathrm{ha}^{-1} \mathrm{año}^{-1}\right)$ asociada a la pérdida de $\mathrm{cm}$ de suelo en el sitio $i$, a: pérdida teórica de rendimiento por erosión por cultivo $\left(\mathrm{kg} \mathrm{ha}^{-1} \mathrm{~cm}^{-1}\right)$ y b: pérdida de suelos por erosión del área de interés $i\left(\mathrm{~cm}\right.$ año $\left.{ }^{-1}\right)$.

Los valores concernientes al factor a, fueron establecidos de acuerdo a estimaciones para la RPArg y consultas a expertos en la temática. En lo referente al factor $b$, los datos se obtuvieron mediante el cociente entre el valor de A (correspondiente al promedio entre los valores de A alcanzados por las dos series de suelo más representativas del área de estudio: Lpd y Ne) y la densidad aparente media (D) del área agrícola de la RPArg (Gaitán et al., 2017). Una vez 
alcanzados los valores de $\Delta \mathrm{y}_{\mathrm{i}}$ de los diversos cultivos, estos se extrapolaron a la superficie sembrada con cada uno de ellos, para las distintas campañas en análisis, según fuentes oficiales (DAA, 2020).

Como último paso, para estimar la pérdida en términos económicos, los resultados de $\Delta y_{\mathrm{i}}$ fueron multiplicados por la cotización de mercado en dólares (USD) de cada uno de los granos (Bolsa de Cereales, 2020), a través de la Ecuación 3, adaptada de Cotler, López y Martínez-Trinidad (2011)

$$
C_{\mathrm{i}}=P_{\mathrm{m}} * \Delta y_{\mathrm{i}}
$$

Donde: $\mathrm{C}_{\mathrm{i}}$ : costo económico de la erosión en el sitio estudiado $i, \mathrm{P}_{\mathrm{m}}$ precio de mercado por th de producto y $\Delta \mathrm{y}_{\mathrm{i}}$ : disminución real del rendimiento $\left(\mathrm{kg} \mathrm{ha}^{-1}\right.$ año $\left.{ }^{-1}\right)$ asociada a la pérdida de $\mathrm{cm}$ de suelo en el sitio $i$.

La totalidad de las ecuaciones presentadas en ambas etapas metodológicas fueron aplicadas en dos oportunidades, considerando distintos escenarios. Por un lado, el escenario ocurrido (I) representa los cálculos realizados teniendo en cuenta la realidad de las prácticas agrícolas efectuadas en el área de estudio; en la cual los valores del factor $\mathrm{P}$, al no haberse concretado ninguna práctica conservacionista, fue siempre igual a 1 . Por su parte, el escenario posible (II) escenifica los resultados que podrían obtenerse en el caso de desarrollarse estrategias tendientes a la conservación del suelo.

\section{Resultados}

Estimación de A, EP y pérdida total de suelos y su variación temporal

Los suelos que componen las UE, debido a sus características, exhiben diversos grados de susceptibilidad a la erosión hídrica. En este sentido, para lograr llevar a cabo la estimación de A y EP, fue indispensable poseer conocimiento acerca de cada uno de los factores componentes de la ecuación. A partir de ello, las tasas de erosión fueron calculadas para cada una de las series de suelo que componen cada UE.

En relación al factor $\mathrm{R}$, se obtuvo considerando el dominio edáfico que caracteriza el área (dominio 13), alcanzando un valor de $318 \mathrm{hJ} \mathrm{cm} \mathrm{m}^{-2}$ $\mathrm{h}^{-1}$ (Irurtia \& Cruzate, 2002). 
Con respecto al factor $\mathrm{K}$, el cual representa la susceptibilidad de los suelos a la erosión hídrica, fue obtenido para cada una de las series de suelo analizadas por UE, luego de aplicada la ecuación correspondiente (Tabla 2).

Tabla 2. Susceptibilidad de los suelos a la erosión hídrica (factor K) por serie de suelo

\begin{tabular}{|c|c|c|c|c|c|c|c|}
\hline UE & Serie & $\begin{array}{l}\text { MO } \\
(\%)\end{array}$ & $\begin{array}{l}\text { Limo +arena } \\
\text { muy fina }(\%)\end{array}$ & $\begin{array}{l}\text { Grado de } \\
\text { Estructura }\end{array}$ & $\begin{array}{l}\text { Permeabilidad } \\
\text { (cm/hora) }\end{array}$ & $\begin{array}{c}\text { Arcilla } \\
(\%)\end{array}$ & $\begin{array}{c}K \\
\left(t \mathrm{~m}^{2} h h^{-1}\right. \\
\left.h^{-1} \mathbf{c m}^{-1}\right) \\
\end{array}$ \\
\hline \multirow[t]{2}{*}{ UE1 } & Lpd & 4,48 & 41,5 & $\begin{array}{c}4 \text { (bloques } \\
\text { subangulares } \\
\text { medios) }\end{array}$ & $\begin{array}{c}3 \text { (moderada: } 2 \\
\text { a } 6,25)\end{array}$ & 25,1 & 0,279 \\
\hline & $\mathrm{Ne}$ & 6,45 & 28,9 & $\begin{array}{c}3 \text { (granular } \\
\text { gruesa) }\end{array}$ & $\begin{array}{c}3 \text { (moderada: } 2 \\
\text { a } 6,25) \\
\end{array}$ & 20,3 & 0,145 \\
\hline \multirow{6}{*}{ UE2 } & Lpd & 4,48 & 41,5 & $\begin{array}{c}4 \text { (bloques } \\
\text { subangulares } \\
\text { medios) }\end{array}$ & $\begin{array}{c}3 \text { (moderada: } 2 \\
\text { a } 6,25)\end{array}$ & 25,1 & 0,279 \\
\hline & $\mathrm{Ne}$ & 6,45 & 28,9 & $\begin{array}{l}3 \text { (granular } \\
\text { gruesa) }\end{array}$ & $\begin{array}{c}3 \text { (moderada: } 2 \\
\text { a } 6,25)\end{array}$ & 20,3 & 0,145 \\
\hline & TA & 3,87 & 26,9 & $\begin{array}{c}3 \text { (granular } \\
\text { gruesa) }\end{array}$ & $\begin{array}{c}4 \text { (moderada- } \\
\text { mente lenta: } 0,5 \\
\text { a } 2)\end{array}$ & 25,9 & 0,202 \\
\hline & Och & 2,74 & 10,1 & 2 (granular fina) & $\begin{array}{c}2 \text { (moderada- } \\
\text { mente rápida: } \\
6,25 \text { a } 12,5)\end{array}$ & 20,8 & 0,019 \\
\hline & $\mathrm{LPg}$ & 6,79 & 29,7 & $\begin{array}{c}3 \text { (granular } \\
\text { gruesa) }\end{array}$ & $\begin{array}{c}3 \text { (moderada: } 2 \\
\text { a } 6,25)\end{array}$ & 18,1 & 0,144 \\
\hline & $\mathrm{Me}$ & 4,80 & 29,1 & $\begin{array}{c}4 \text { (bloques } \\
\text { subangulares } \\
\text { medios) }\end{array}$ & $\begin{array}{c}5 \text { (lenta: } 0,125 \text { a } \\
0,500)\end{array}$ & 20,6 & 0,282 \\
\hline \multirow{4}{*}{ UE3 } & Lo & 5,59 & 37 & $\begin{array}{l}3 \text { (granular } \\
\text { gruesa) }\end{array}$ & $\begin{array}{l}4 \text { (moderadamen- } \\
\text { te lenta: } 0,5 \text { a } 2 \text { ) }\end{array}$ & 30,1 & 0,209 \\
\hline & Lpd & 4,48 & 41,5 & $\begin{array}{c}4 \text { (bloques } \\
\text { subangulares } \\
\text { medios) }\end{array}$ & $\begin{array}{c}3 \text { (moderada: } 2 \\
\text { a } 6,25)\end{array}$ & 25,1 & 0,279 \\
\hline & $\mathrm{Ne}$ & 6,45 & 28,9 & $\begin{array}{l}3 \text { (granular } \\
\text { gruesa) }\end{array}$ & $\begin{array}{c}3 \text { (moderada: } 2 \\
\text { a } 6,25)\end{array}$ & 20,3 & 0,145 \\
\hline & $\mathrm{Me}$ & 4,80 & 29,1 & $\begin{array}{l}4 \text { (bloques } \\
\text { subangulares } \\
\text { medios) }\end{array}$ & $\begin{array}{c}5 \text { (lenta: } 0,125 \text { a } \\
0,500)\end{array}$ & 20,6 & 0,282 \\
\hline
\end{tabular}

Referencias: Lpd: Laprida, Ne: Necochea, TA: Tres Arroyos, Och: Ochandio, LPg: La Pandorga, Me: Mechongué, Lo: Lobería.

Fuente: Elaboración propia. 
A continuación, los datos requeridos para la obtención del factor LS, el cual tiene en consideración la longitud de las pendientes y su gradiente, fueron estimados en función de los suelos dominantes en cada unidad, alcanzándose los resultados expresados en la Tabla 3.

Tabla 3. Longitud de las pendientes y gradiente (factor LS) por serie de suelo

\begin{tabular}{|c|c|c|c|c|}
\hline UE & Serie & $\begin{array}{l}\text { Longitud de la pendiente } \\
\text { (m) }\end{array}$ & $\begin{array}{l}\text { Pendiente } \\
(\%)\end{array}$ & $\mathbf{L S}$ \\
\hline \multirow{2}{*}{ UE1 } & Lpd & 200 & 1 & 0,19 \\
\hline & $\mathrm{Ne}$ & 200 & 1 & 0,19 \\
\hline \multirow{6}{*}{ UE2 } & Lpd & 200 & 1 & 0,19 \\
\hline & $\mathrm{Ne}$ & 200 & 1 & 0,19 \\
\hline & TA & 200 & 2 & 0,42 \\
\hline & Och & 200 & 1 & 0,19 \\
\hline & $\mathrm{LPg}$ & 200 & 0,75 & 0,14 \\
\hline & $\mathrm{Me}$ & 200 & 0,5 & 0,1 \\
\hline \multirow{4}{*}{ UE3 } & Lo & 200 & 0,5 & 0,1 \\
\hline & Lpd & 200 & 1 & 0,19 \\
\hline & $\mathrm{Ne}$ & 200 & 1 & 0,19 \\
\hline & $\mathrm{Me}$ & 200 & 0,5 & 0,1 \\
\hline
\end{tabular}

Referencias: Lpd: Laprida, Ne: Necochea, TA: Tres Arroyos, Och: Ochandio, LPg: La Pandorga, Me: Mechongué, Lo: Lobería.

Fuente: Elaboración propia.

El valor adquirido para el factor $\mathrm{C}$, fue variable conforme a la época considerada. Al calcularlo, la diferencia de mayor relevancia se debió a que, en la campaña 1989/90, la agricultura dentro del área de estudio era desarrollada mediante la denominada Siembra Convencional (SC) o tradicional. No obstante, en oposición a lo anterior, tanto para las campañas 2002/2003 y 2019/2020, la SC fue reemplazada por SD, casi en la totalidad de la superficie. En base a ello, el valor de C para 1989/90 fue de 0,171 y surgió de un promedio entre los valores de $\mathrm{C}$ correspondientes a los planteos "Maíz en rotación”, "Trigo en rotación” y "Soja en rotación".

En cuanto a 2002/03 y 2019/20, se llevó a cabo un promedio entre los valores de los planteos "Maíz en rotación", "Trigo/soja de segunda" y "Soja continua", representando el factor C un valor igual a 0,089. En este sentido, cabe destacar que los planteos productivos elegidos para la 
obtención de este factor, se corresponden a los mayormente empleados dentro del área de estudio en las distintas campañas.

Por último, los valores concernientes al factor P presentaron diferencias, teniendo en cuenta los dos escenarios planteados. En el caso del escenario I, el valor asciende a 1 en la totalidad de las campañas, ya que no se registró la aplicación de ninguna práctica conservacionista. Para el escenario II, en la campaña 1989/90, el valor de P se estimó considerando la realización de estrategias como "siembra en contorno y labranza convencional", lo cual arrojó un valor igual a 0,5 ; mientras que para 2002/03 y 2019/20, al considerar las estrategias "terrazas no sembrables y siembra en contorno", $P$ fue igual a 0,4 .

El resultado del producto de los factores mencionados anteriormente $\left(\mathrm{R}^{*} \mathrm{~K}^{*} \mathrm{LS} \mathrm{C}^{*} \mathrm{P}\right)$ facilitó el cálculo de A para cada una de las series de suelo analizadas. Por otro lado, la estimación de EP surgió de igual forma que A, pero llevando a cabo únicamente el producto de los factores R, K y LS (sin considerar C ni P). Seguidamente, las Tablas 4 y 5 demuestran los valores alcanzados para A y EP en las series correspondientes a las unidades sobre las que son desarrolladas las actividades agrícolas del partido en el escenario I; mientras que, las Tablas 6 y 7 hacen lo propio para el escenario II.

Tabla 4. Erosión actual (A) y potencial (EP) para la campaña 1989/90, escenario I

\begin{tabular}{|c|c|c|c|c|c|c|c|c|}
\hline UE & Serie & $\mathbf{R}$ & $\mathbf{K}$ & $\mathbf{L S}$ & $\mathbf{C}$ & $\mathbf{P}$ & $\mathbf{A}$ & $\mathbf{E P}$ \\
\hline \multirow{3}{*}{ UE1 } & Lpd & 318 & 0,279 & 0,19 & 0,171 & 1 & 2,9 & 16,86 \\
\cline { 2 - 9 } & $\mathrm{Ne}$ & 318 & 0,145 & 0,19 & 0,171 & 1 & 1,5 & 8,76 \\
\hline \multirow{4}{*}{ UE2 } & Lpd & 318 & 0,279 & 0,19 & 0,171 & 1 & 2,9 & 16,86 \\
\cline { 2 - 9 } & $\mathrm{Ne}$ & 318 & 0,145 & 0,19 & 0,171 & 1 & 1,5 & 8,76 \\
\cline { 2 - 9 } & $\mathrm{TA}$ & 318 & 0,202 & 0,42 & 0,171 & 1 & 4,6 & 26,98 \\
\cline { 2 - 9 } & $\mathrm{Och}$ & 318 & 0,019 & 0,19 & 0,171 & 1 & 0,2 & 1,15 \\
\cline { 2 - 9 } & $\mathrm{LPg}$ & 318 & 0,144 & 0,14 & 0,171 & 1 & 1,1 & 6,41 \\
\cline { 2 - 9 } & $\mathrm{Me}$ & 318 & 0,282 & 0,1 & 0,171 & 1 & 1,5 & 8,97 \\
\hline \multirow{4}{*}{ UE3 } & $\mathrm{Lo}$ & 318 & 0,209 & 0,1 & 0,171 & 1 & 1,1 & 6,65 \\
\cline { 2 - 9 } & $\mathrm{Lpd}$ & 318 & 0,279 & 0,19 & 0,171 & 1 & 2,9 & 16,86 \\
\cline { 2 - 9 } & $\mathrm{Ne}$ & 318 & 0,145 & 0,19 & 0,171 & 1 & 1,5 & 8,76 \\
\cline { 2 - 9 } & $\mathrm{Me}$ & 318 & 0,282 & 0,1 & 0,171 & 1 & 1,5 & 8,97 \\
\hline
\end{tabular}

Referencias: Lpd: Laprida, Ne: Necochea, TA: Tres Arroyos, Och: Ochandio, LPg: La Pandorga, Me: Mechongué, Lo: Lobería, R: Erosividad de las lluvias $\left(\mathrm{hJ} \mathrm{cm} \mathrm{m}^{-2} \mathrm{~h}^{-1}\right), \mathrm{K}$ : Susceptibilidad del suelo a la erosión hídrica $\left(\mathrm{t} \mathrm{m}^{2} \mathrm{~h} \mathrm{ha}^{-1} \mathrm{hJ}^{-1} \mathrm{~cm}^{-1}\right)$, LS: Factor topográfico, C: Factor cultivo o cobertura, P: Factor práctica conservacionista, A: Tasa de erosión actual $\left(\mathrm{t} \mathrm{ha}^{-1} \mathrm{año}^{-1}\right)$ y EP: Tasa de erosión potencial $\left(\mathrm{t} \mathrm{ha} \mathrm{a}^{-1}\right.$ añ $\left.{ }^{-1}\right)$.

Fuente: Elaboración propia. 
Nahuel David Sequeira - Patricia Vazquez

Impacto de la erosión hídrica sobre la rentabilidad de los productores agrícolas en el partido

de Tres Arroyos, Región Pampeana Austral, Argentina

Tabla 5. Erosión actual (A) y potencial (EP) para las campañas 2002/03 y $2019 / 20$, escenario I

\begin{tabular}{|c|c|c|c|c|c|c|c|c|}
\hline UE & Serie & $\mathbf{R}$ & $\mathbf{K}$ & $\mathbf{L S}$ & $\mathbf{C}$ & $\mathbf{P}$ & $\mathbf{A}$ & $\mathbf{E P}$ \\
\hline \multirow{2}{*}{ UE1 } & Lpd & 318 & 0,279 & 0,19 & 0,089 & 1 & 1,5 & 16,86 \\
\cline { 2 - 9 } & $\mathrm{Ne}$ & 318 & 0,145 & 0,19 & 0,089 & 1 & 0,8 & 8,76 \\
\hline \multirow{4}{*}{ UE2 } & Lpd & 318 & 0,279 & 0,19 & 0,089 & 1 & 1,5 & 16,86 \\
\cline { 2 - 9 } & $\mathrm{Ne}$ & 318 & 0,145 & 0,19 & 0,089 & 1 & 0,8 & 8,76 \\
\cline { 2 - 9 } & $\mathrm{TA}$ & 318 & 0,202 & 0,42 & 0,089 & 1 & 2,4 & 26,98 \\
\cline { 2 - 9 } & Och & 318 & 0,019 & 0,19 & 0,089 & 1 & 0,1 & 1,15 \\
\cline { 2 - 9 } & LPg & 318 & 0,144 & 0,14 & 0,089 & 1 & 0,6 & 6,41 \\
\cline { 2 - 9 } & $\mathrm{Me}$ & 318 & 0,282 & 0,1 & 0,089 & 1 & 0,8 & 8,97 \\
\hline \multirow{4}{*}{ UE3 } & Lo & 318 & 0,209 & 0,1 & 0,089 & 1 & 0,6 & 6,65 \\
\cline { 2 - 9 } & $\mathrm{Lpd}$ & 318 & 0,279 & 0,19 & 0,089 & 1 & 1,5 & 16,86 \\
\cline { 2 - 9 } & $\mathrm{Ne}$ & 318 & 0,145 & 0,19 & 0,089 & 1 & 0,8 & 8,76 \\
\cline { 2 - 9 } & $\mathrm{Me}$ & 318 & 0,282 & 0,1 & 0,089 & 1 & 0,8 & 8,97 \\
\hline
\end{tabular}

Referencias: Lpd: Laprida, Ne: Necochea, TA: Tres Arroyos, Och: Ochandio, LPg: La Pandorga, Me: Mechongué, Lo: Lobería, R: Erosividad de las lluvias $\left(\mathrm{hJ} \mathrm{cm} \mathrm{m}^{-2} \mathrm{~h}^{-1}\right), \mathrm{K}$ : Susceptibilidad del suelo a la erosión hídrica $\left(\mathrm{t} \mathrm{m}^{2} \mathrm{~h} \mathrm{ha}^{-1} \mathrm{hJ}^{-1} \mathrm{~cm}^{-1}\right)$, LS: Factor topográfico, C: Factor cultivo o cobertura, P: Factor práctica conservacionista, A: Tasa de erosión actual $\left(\mathrm{t} \mathrm{ha}^{-1} \mathrm{año}^{-1}\right)$ y EP: Tasa de erosión potencial $\left(\mathrm{t} \mathrm{ha} \mathrm{añ}^{-1} \mathrm{an}^{-1}\right)$.

Fuente: Elaboración propia.

Tabla 6. Erosión actual (A) y potencial (EP) para la campaña 1989/90, escenario II

\begin{tabular}{|c|c|c|c|c|c|c|c|c|}
\hline UE & Serie & $\mathbf{R}$ & $\mathbf{K}$ & LS & $\mathbf{C}$ & $\mathbf{P}$ & $\mathbf{A}$ & $\mathbf{E P}$ \\
\hline UE1 & Lpd & 318 & 0,279 & 0,19 & 0,171 & 0,5 & 1,4 & 16,86 \\
\hline & $\mathrm{Ne}$ & 318 & 0,145 & 0,19 & 0,171 & 0,5 & 0,8 & 8,76 \\
\hline UE2 & Lpd & 318 & 0,279 & 0,19 & 0,171 & 0,5 & 1,4 & 16,86 \\
\hline & $\mathrm{Ne}$ & 318 & 0,145 & 0,19 & 0,171 & 0,5 & 0,8 & 8,76 \\
\hline & TA & 318 & 0,202 & 0,42 & 0,171 & 0,5 & 2,3 & 26,98 \\
\hline & Och & 318 & 0,019 & 0,19 & 0,171 & 0,5 & 0,1 & 1,15 \\
\hline & LPg & 318 & 0,144 & 0,14 & 0,171 & 0,5 & 0,6 & 6,41 \\
\hline & Me & 318 & 0,282 & 0,1 & 0,171 & 0,5 & 0,8 & 8,97 \\
\hline UE3 & Lo & 318 & 0,209 & 0,1 & 0,171 & 0,5 & 0,6 & 6,65 \\
\hline & Lpd & 318 & 0,279 & 0,19 & 0,171 & 0,5 & 1,4 & 16,86 \\
\hline & Ne & 318 & 0,145 & 0,19 & 0,171 & 0,5 & 0,8 & 8,76 \\
\hline & Me & 318 & 0,282 & 0,1 & 0,171 & 0,5 & 0,8 & 8,97 \\
\hline
\end{tabular}

Referencias: Lpd: Laprida, Ne: Necochea, TA: Tres Arroyos, Och: Ochandio, LPg: La Pandorga, Me: Mechongué, Lo: Lobería, R: Erosividad de las lluvias $\left(\mathrm{hJ} \mathrm{cm} \mathrm{m}^{-2} \mathrm{~h}^{-1}\right), \mathrm{K}$ : Susceptibilidad del suelo a la erosión hídrica $\left(\mathrm{t} \mathrm{m}^{2} \mathrm{~h} \mathrm{ha}^{-1} \mathrm{hJ}^{-1} \mathrm{~cm}^{-1}\right)$, LS: Factor topográfico, C: Factor cultivo o cobertura, P: Factor práctica conservacionista, A: Tasa de erosión actual $\left(\mathrm{t} \mathrm{ha}^{-1} \mathrm{año}^{-1}\right)$ y EP: Tasa de erosión potencial ( $\mathrm{t} \mathrm{ha} \mathrm{ann}^{-1}$ ).

Fuente: Elaboración propia. 
Tabla 7. Erosión actual (A) y potencial (EP) para las campañas 2002/03 y $2019 / 20$, escenario II

\begin{tabular}{|c|c|c|c|c|c|c|c|c|}
\hline UE & Serie & $\mathbf{R}$ & $\mathbf{K}$ & $\mathbf{L S}$ & $\mathbf{C}$ & $\mathbf{P}$ & $\mathbf{A}$ & $\mathbf{E P}$ \\
\hline \multirow{3}{*}{ UE1 } & Lpd & 318 & 0,279 & 0,19 & 0,089 & 0,4 & 0,6 & 16,86 \\
\cline { 2 - 9 } & $\mathrm{Ne}$ & 318 & 0,145 & 0,19 & 0,089 & 0,4 & 0,3 & 8,76 \\
\hline \multirow{4}{*}{ UE2 } & Lpd & 318 & 0,279 & 0,19 & 0,089 & 0,4 & 0,6 & 16,86 \\
\cline { 2 - 9 } & $\mathrm{Ne}$ & 318 & 0,145 & 0,19 & 0,089 & 0,4 & 0,3 & 8,76 \\
\cline { 2 - 9 } & $\mathrm{TA}$ & 318 & 0,202 & 0,42 & 0,089 & 0,4 & 1 & 26,98 \\
\cline { 2 - 9 } & Och & 318 & 0,019 & 0,19 & 0,089 & 0,4 & 0,1 & 1,15 \\
\cline { 2 - 9 } & LPg & 318 & 0,144 & 0,14 & 0,089 & 0,4 & 0,2 & 6,41 \\
\cline { 2 - 9 } & $\mathrm{Me}$ & 318 & 0,282 & 0,1 & 0,089 & 0,4 & 0,3 & 8,97 \\
\hline \multirow{4}{*}{ UE3 } & Lo & 318 & 0,209 & 0,1 & 0,089 & 0,4 & 0,2 & 6,65 \\
\cline { 2 - 9 } & $\mathrm{Lpd}$ & 318 & 0,279 & 0,19 & 0,089 & 0,4 & 0,6 & 16,86 \\
\cline { 2 - 9 } & $\mathrm{Ne}$ & 318 & 0,145 & 0,19 & 0,089 & 0,4 & 0,3 & 8,76 \\
\cline { 2 - 9 } & $\mathrm{Me}$ & 318 & 0,282 & 0,1 & 0,089 & 0,4 & 0,3 & 8,97 \\
\hline
\end{tabular}

Referencias: Lpd: Laprida, Ne: Necochea, TA: Tres Arroyos, Och: Ochandio, LPg: La Pandorga, Me: Mechongué, Lo: Lobería, R: Erosividad de las lluvias $\left(\mathrm{hJ} \mathrm{cm} \mathrm{m}^{-2} \mathrm{~h}^{-1}\right)$, K: Susceptibilidad del suelo a la erosión hídrica $\left(\mathrm{t} \mathrm{m}^{2} \mathrm{~h} \mathrm{ha}^{-1} \mathrm{hJ}^{-1} \mathrm{~cm}^{-1}\right)$, LS: Factor topográfico, C: Factor cultivo o cobertura, P: Factor práctica conservacionista, A: Tasa de erosión actual $\left(\mathrm{t} \mathrm{ha}^{-1} \mathrm{año}^{-1}\right)$ y EP: Tasa de erosión potencial $\left(\mathrm{t} \mathrm{ha}{ }^{-1} \mathrm{año}^{-1}\right)$.

Fuente: Elaboración propia.

Sumado a lo anterior, se efectuó un análisis detallado acerca de cómo varió el valor de A para cada serie de suelo, entre las distintas campañas para el escenario I (Figura 2) y el escenario II (Figura 3); como así también acerca de la modificación de este valor de erosión para cada serie en una misma campaña, según el escenario propuesto (Figuras 4 y 5). 
Figura 2. Variación de la tasa de erosión actual (A) por serie en las distintas campañas, para el escenario I

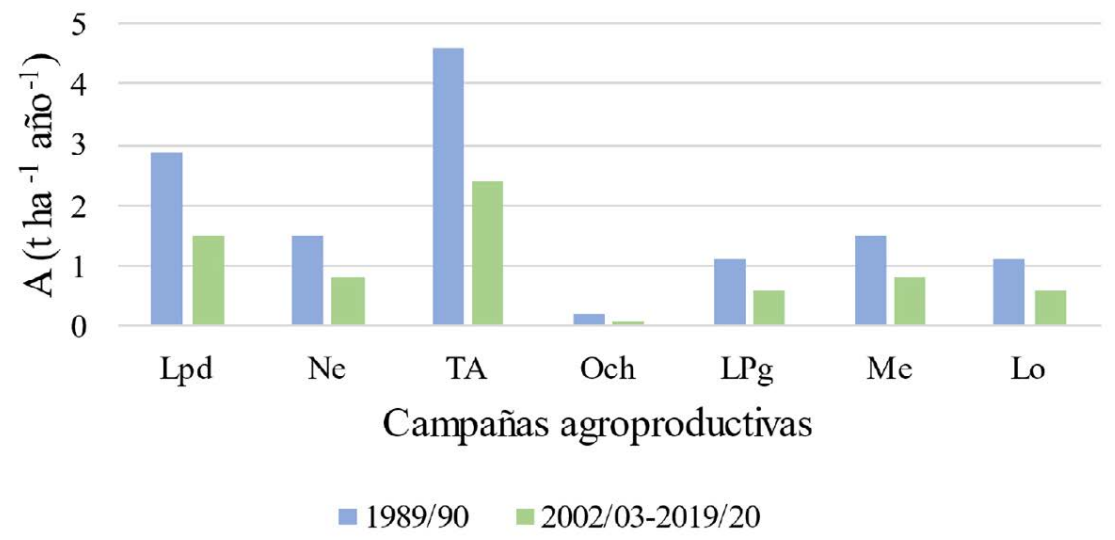

Referencias: Lpd: Laprida, Ne: Necochea, TA: Tres Arroyos, Och: Ochandio, LPg: La Pandorga, Me: Mechongué, Lo: Lobería, A: Tasa de erosión anual.

Fuente: Elaboración propia.

Figura 3. Variación de la tasa de erosión actual (A) por serie en las distintas campañas, para el escenario II

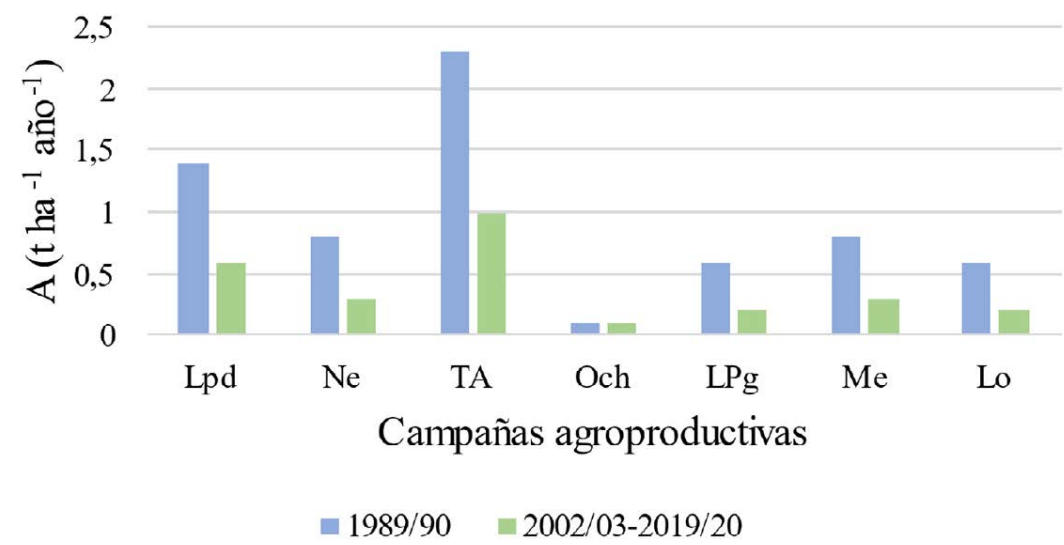

Referencias: Lpd: Laprida, Ne: Necochea, TA: Tres Arroyos, Och: Ochandio, LPg: La Pandorga, Me: Mechongué, Lo: Lobería, A: Tasa de erosión anual.

Fuente: Elaboración propia. 
Figura 4. Variación de la tasa de erosión actual por serie en ambos escenarios, para la campaña 1989/90

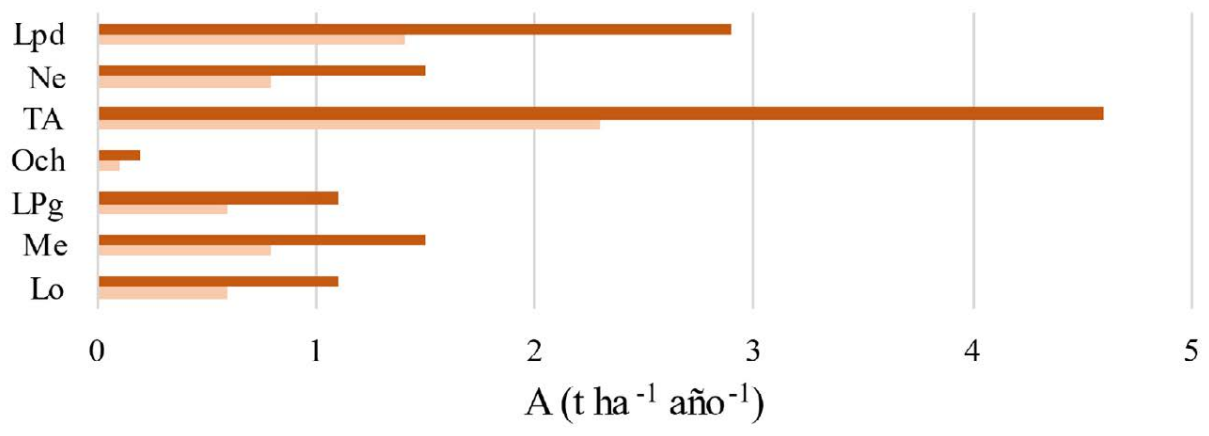

- Escenario I $\quad$ Escenario II

Referencias: Lpd: Laprida, Ne: Necochea, TA: Tres Arroyos, Och: Ochandio, LPg: La Pandorga, Me: Mechongué, Lo: Lobería, A: Tasa de erosión anual.

Fuente: Elaboración propia.

Figura 5. Variación de la tasa de erosión actual por serie en ambos escenarios, para las campañas 2002/03 y 2019/20

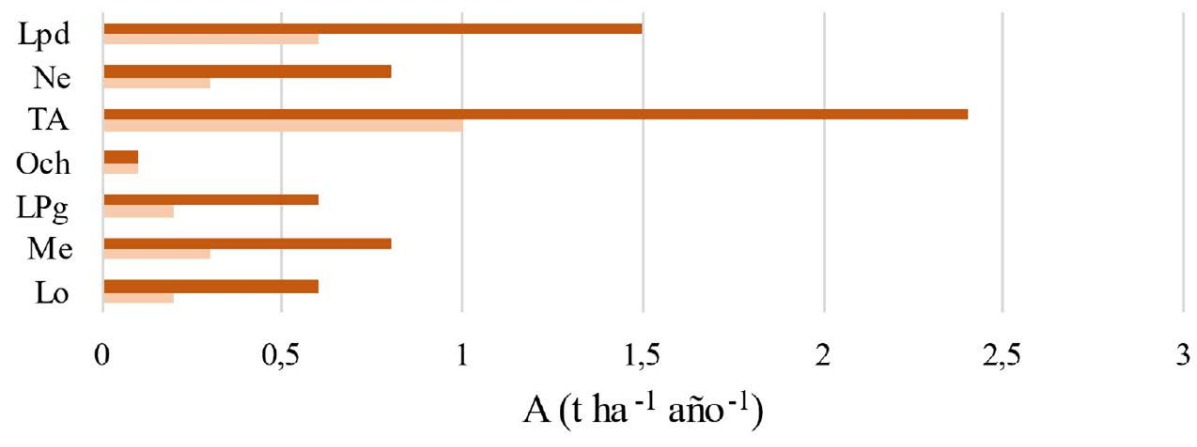

- Escenario I $=$ Escenario II

Referencias: Lpd: Laprida, Ne: Necochea, TA: Tres Arroyos, Och: Ochandio, LPg: La Pandorga, Me: Mechongué, Lo: Lobería, A: Tasa de erosión anual.

Fuente: Elaboración propia. 
Con respecto a la pérdida de suelos, se seleccionaron las dos series más representativas de las tres unidades ( $\mathrm{Lpd}$ y Ne) y se llevó a cabo un promedio de su A y EP, obteniendo distintos valores para ambos escenarios, los cuales se representan en la Tabla 8.

Tabla 8. Promedio de las tasas de erosión para los dos escenarios analizados

\begin{tabular}{|c|c|c|c|c|}
\hline \multirow{3}{*}{$\begin{array}{l}\text { Valores promedio de erosión entre } \\
\text { Lpd y Ne } \\
\left(\mathrm{t} \mathrm{ha}^{-1} \mathrm{año}^{-1}\right)\end{array}$} & \multicolumn{2}{|c|}{ Escenario I } & \multicolumn{2}{|c|}{ Escenario II } \\
\hline & \multicolumn{4}{|c|}{ Campañas agroproductivas } \\
\hline & $1989 / 90$ & $\begin{array}{l}2002 / 03 \\
2019 / 20\end{array}$ & $1989 / 90$ & $\begin{array}{l}2002 / 03 \\
2019 / 20\end{array}$ \\
\hline Promedio de A & 2,2 & 1,15 & 1,1 & 0,45 \\
\hline Promedio de EP & 12,81 & 12,81 & 12,81 & 12,81 \\
\hline
\end{tabular}

Referencias: Lpd: Laprida, Ne: Necochea, A: Tasa de erosión actual ( $\left.\mathrm{t} \mathrm{ha}^{-1} \mathrm{año}^{-1}\right)$ y EP: Tasa de erosión potencial ( $\left.\mathrm{t} \mathrm{ha} \mathrm{h}^{-1} \mathrm{año}^{-1}\right)$.

Fuente: Elaboración propia.

Finalmente, los promedios alcanzados se multiplicaron por la superficie agrícola destinada a tres de los principales cultivos del partido para ambos escenarios en 1989/90, 2002/03 y 2019/20, adquiriendo de esta manera los valores concernientes a la PTSA y PTSP, tal como se muestra mediante la Tabla 9.

Tabla 9. Pérdida total de suelos por campaña en ambos escenarios

\begin{tabular}{|c|c|c|c|c|c|}
\hline \multirow{2}{*}{$\begin{array}{c}\text { Campañas } \\
\text { agroproductivas }\end{array}$} & \multirow{2}{*}{$\begin{array}{c}\text { Superficie } \\
\text { agrícola (ha) }\end{array}$} & \multicolumn{2}{|c|}{ Escenario I } & \multicolumn{2}{|c|}{ Escenario II } \\
\hline & & $\begin{array}{c}\text { PTSA } \\
\left(\text { t } \mathbf{a n ̃ o} o^{-1}\right)\end{array}$ & $\begin{array}{c}\text { PTSP } \\
(t \text { año-1 })\end{array}$ & $\begin{array}{c}\text { PTSA } \\
\left(\text { t } \mathbf{a n ̃ o} 0^{-1}\right)\end{array}$ & $\begin{array}{c}\text { PTSP } \\
(\text { t año-1) }\end{array}$ \\
\hline $1989 / 90$ & 201000 & 442200 & 2574810 & 221100 & 2574810 \\
\hline $2002 / 03$ & 279000 & 320850 & 3573990 & 125550 & 3573990 \\
\hline $2019 / 20$ & 323000 & 371450 & 4137630 & 145350 & 4137630 \\
\hline
\end{tabular}

Referencias: PTSA: Pérdida total de suelos actual y PTSP: Pérdida total de suelos potencial.

Fuente: Elaboración propia.

\section{Análisis acerca de la pérdida económica}

Una vez concretados los cálculos, se muestran en las Tablas 10 y 11 los valores de los diversos factores, analizados para ambos escenarios. A partir de ello, fue factible desarrollar la estimación de la disminución del rendimiento de tres de los principales cultivos del partido, para las tres campañas evaluadas, observándose los datos obtenidos en las Tablas 12 y 13. 
Tabla 10. Valores de los diversos factores analizados en el escenario I

\begin{tabular}{|c|c|c|c|c|c|c|c|}
\hline \multirow{5}{*}{$\begin{array}{l}\text { partido de } \\
\text { Tres Arroyos }\end{array}$} & \multirow[b]{2}{*}{ Cultivo } & \multirow{2}{*}{$\frac{\mathrm{a}}{\left(\mathrm{kg} \mathrm{ha}^{-1} \mathrm{~cm}^{-1}\right)}$} & \multirow{2}{*}{$\begin{array}{c}D \\
\left(t_{~ m^{-3}}\right)\end{array}$} & \multicolumn{2}{|c|}{ TME $\left(t\right.$ ha $\left.^{-1} a_{\tilde{n}} 0^{-1}\right)$} & \multicolumn{2}{|c|}{ b $\left(\mathbf{c m}\right.$ año $\left.{ }^{-1}\right)$} \\
\hline & & & & $1989 / 90$ & $\begin{array}{l}2002 / 03 \\
2019 / 20\end{array}$ & $1989 / 90$ & $\begin{array}{l}2002 / 03 \\
2019 / 20\end{array}$ \\
\hline & Soja & 100 & \multirow{3}{*}{1,2} & \multirow{3}{*}{2,2} & \multirow{3}{*}{1,15} & \multirow{3}{*}{0,018} & \multirow{3}{*}{0,009} \\
\hline & Maíz & 270 & & & & & \\
\hline & Trigo & 75 & & & & & \\
\hline
\end{tabular}

Referencias: a: pérdida teórica de rendimiento por erosión para cada cultivo, D: densidad aparente media del suelo agrícola; TME: tasa media de erosión hídrica y B: pérdida de suelos por erosión del área de interés.

Fuente: Elaboración propia.

Tabla 11. Valores de los diversos factores analizados en el escenario II

\begin{tabular}{|c|c|c|c|c|c|c|c|}
\hline \multirow{5}{*}{$\begin{array}{l}\text { partido de } \\
\text { Tres Arroyos }\end{array}$} & \multirow[b]{2}{*}{ Cultivo } & \multirow{2}{*}{$\begin{array}{c}\mathrm{a} \\
\left(\mathrm{kg} \mathrm{ha}^{-1} \mathrm{~cm}^{-1}\right)\end{array}$} & \multirow{2}{*}{$\underset{\left(t ~ m^{-3}\right)}{D}$} & \multicolumn{2}{|c|}{ TME $\left._{\left(t ~ h a^{-1}\right.} a^{-1} 0^{-1}\right)$} & \multicolumn{2}{|c|}{ b $(\mathbf{c m}$ año-1) } \\
\hline & & & & 1989/90 & $\begin{array}{l}2002 / 03 \\
2019 / 20\end{array}$ & 1989/90 & $\begin{array}{l}2002 / 03 \\
2019 / 20\end{array}$ \\
\hline & Soja & 100 & \multirow[t]{3}{*}{1,2} & \multirow[t]{3}{*}{1,1} & \multirow[t]{3}{*}{0,45} & \multirow[t]{3}{*}{0,009} & \multirow[t]{3}{*}{0,004} \\
\hline & Maíz & 270 & & & & & \\
\hline & Trigo & 75 & & & & & \\
\hline
\end{tabular}

Referencias: a: pérdida teórica de rendimiento por erosión para cada cultivo, D: densidad aparente media del suelo agrícola; TME: tasa media de erosión hídrica y B: pérdida de suelos por erosión del área de interés.Fuente: elaboración propia.

Tabla 12. Disminución real del rendimiento por campaña en el escenario I

\begin{tabular}{|c|c|c|c|}
\hline \multirow{4}{*}{ partido de Tres Arroyos } & \multirow{2}{*}{ Cultivo } & \multicolumn{2}{|c|}{$\mathbf{R}\left(\mathbf{k g ~ h a}^{-1} \mathbf{a n o}^{-\mathbf{1}}\right)$} \\
\cline { 3 - 4 } & Soja & $\mathbf{1 9 8 9 / 9 0}$ & $\mathbf{2 0 0 2 / 0 3 - 2 0 1 9 / 2 0}$ \\
\cline { 2 - 4 } & Maíz & 1,8 & 0,9 \\
\cline { 2 - 4 } & Trigo & 4,86 & 2,43 \\
\cline { 2 - 4 } & 1,35 & 0,68 \\
\hline
\end{tabular}

Referencias: R: disminución real del rendimiento de los cultivos.

Fuente: Elaboración propia.

Tabla 13. Disminución real del rendimiento por campaña en el escenario II

\begin{tabular}{|c|c|c|c|}
\hline \multirow{4}{*}{ partido de Tres Arroyos } & \multirow{2}{*}{ Cultivo } & \multicolumn{2}{|c|}{$\mathbf{R}\left(\mathbf{k g ~ h a}^{-\mathbf{~}} \mathbf{\text { año }} \mathbf{-}^{-1}\right)$} \\
\cline { 2 - 4 } & & $\mathbf{1 9 8 9 / 9 0}$ & $\mathbf{2 0 0 2 / 0 3 - 2 0 1 9 / 2 0}$ \\
\cline { 2 - 4 } & Soja & 0,9 & 0,4 \\
\cline { 2 - 4 } & Maíz & 2,43 & 1,08 \\
\cline { 2 - 4 } & Trigo & 0,68 & 0,30 \\
\hline
\end{tabular}

Referencias: R: disminución real del rendimiento de los cultivos.

Fuente: Elaboración propia. 
Posteriormente, se presentan en las Figuras 6 y 7 los resultados alcanzados respecto de la pérdida total de producción y la concerniente a cada uno de los cultivos, como así también su impacto económico, para las tres campañas en los dos escenarios analizados.

Figura 6. Pérdida de producción e impacto económico en el escenario I

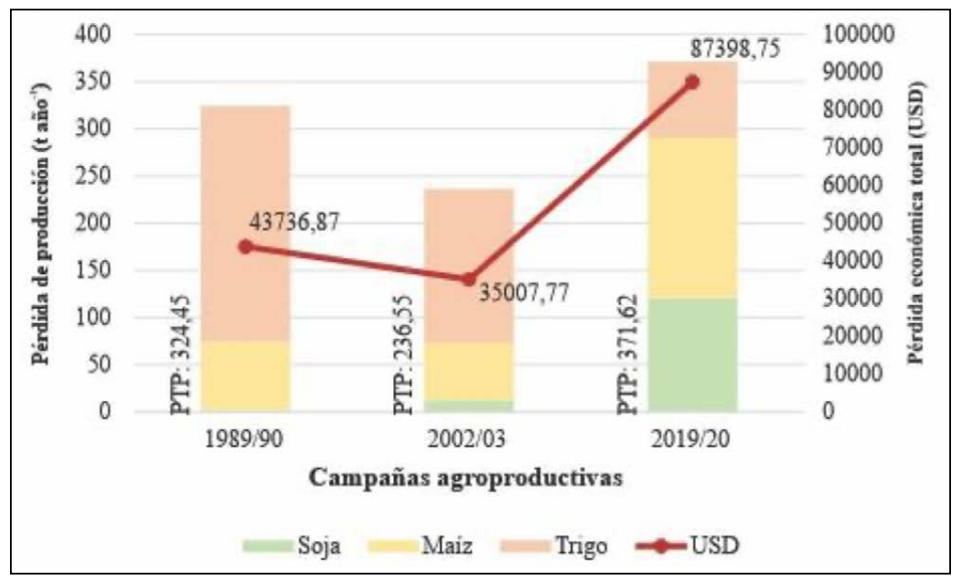

Referencias: PTP: pérdida total de producción $(\mathrm{t}$ año-1 $)$.

Fuente: Elaboración propia.

Figura 7. Pérdida de producción e impacto económico en el escenario II

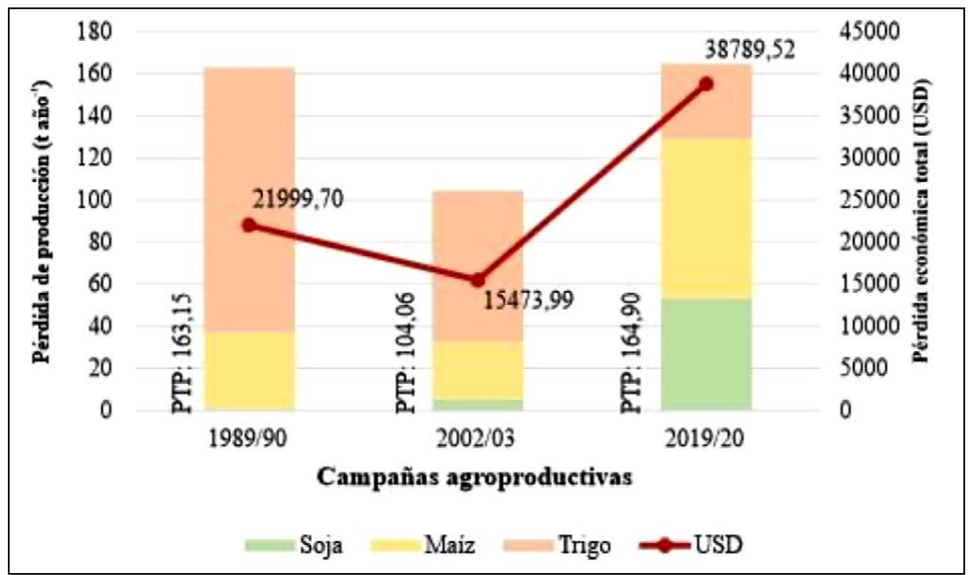

Referencias: PTP: pérdida total de producción $\left(\mathrm{t}\right.$ año $\left.{ }^{-1}\right)$.

Fuente: Elaboración propia. 


\section{Discusión}

\section{Estimación de A, EP, pérdida total de suelos y su variación temporal}

En un primer punto, es necesario destacar que los valores de A adquiridos para las campañas 2002/03 y 2019/20 son los mismos, ya que en ese lapso temporal no se produce en el área ninguna transformación sustancial de las prácticas productivas asociadas al problema de la erosión. Sumado a lo anterior, es posible observar que, como era de esperarse, se obtuvieron los mismos valores de EP para las tres campañas; lo cual puede explicarse debido a que esta es considerada como la máxima posible para un determinado sitio (teniendo en cuenta un suelo desnudo todo el año), razón por la que los valores de los factores relacionados a la cobertura (C) y las prácticas conservacionistas $(\mathrm{P})$ no poseen influencia sobre el valor final.

Una vez analizados los resultados, es viable afirmar la existencia de notables diferencias en cuanto a los valores alcanzados para las distintas series y ambos escenarios. Inicialmente, tanto el escenario I como el II exhiben una tendencia similar respecto a la disminución del valor de A entre las campañas 1989/90-2002/03 y su mantenimiento entre 2002-032019/20, para la totalidad de las series evaluadas; a excepción de la denominada Och, en el escenario II, cuyo valor se mantuvo constante.

Por otra parte, se distingue que para las tres campañas los resultados de A fueron mejores al aplicar prácticas tendientes a la conservación del suelo (escenario II), obteniendo valores con un promedio de disminución aproximada del $50 \%$ en la totalidad de las series.

Respecto de los niveles de afectación, en todos los casos, las series más impactadas resultan ser TA (Tres Arroyos) y Lpd (Laprida). En el escenario I, TA revela valores de A iguales a 4,6 en 1989/90 y 2,4 en 2002/03-2019/20; mientras que Lpd manifiesta valores de 2,9 y 1,5, respectivamente. Para el escenario II los valores de A de TA resultan iguales a 2,3 en 1989/90 y 1 en 2002/03-2019/20 y los de Lpd a 1,4 y 0,6. Asimismo, estas dos series también se presentan como aquellas con mayor valor de EP, con 26,98 y 16,86, respectivamente.

En relación a la pérdida total de suelos, también existen disparidades entre las campañas para ambos escenarios. Para el caso del I, entre $1989 / 90$ y $2002 / 03$ se produjo un decrecimiento del 27,44\% de la PTSA; mientras que, por el contrario, entre 2002/03 y 2019/20, la situación fue 
inversa, con un incremento del 15,77 \%. En cuanto a la PTSP, demuestra un notable incremento en ambos períodos, con valores del $38,80 \%$ entre $1989 / 90$ y $2002 / 03$ y del $15,77 \%$ entre $2002 / 03$ y $2019 / 20$.

Por su parte, aunque la situación del escenario II presenta porcentajes de variación diferentes, queda demostrada exactamente, la misma tendencia. Entre 1989/90 y 2002/03, se produjo un decrecimiento del 43,21\% de la PTSA; y contrariamente, entre 2002/03 y 2019/20, la situación fue inversa, con un incremento del 15,91\%.

Nuevamente, al igual que con los valores de A, queda de manifiesto que al desarrollar estrategias tendientes a la conservación del suelo (escenario II), la situación mejora considerablemente, alcanzando una disminución de aproximadamente el 55\% de la PTSA. Asimismo, al igual que lo que ocurre con la EP, los valores de PTSP no se ven afectados por la aplicación de las mencionadas estrategias.

Independientemente del escenario analizado, una posible explicación de estas notables variaciones, reside en la relación directa entre la erosión hídrica y la superficie destinada a la agricultura. En este sentido, el decrecimiento de la pérdida de suelos entre 1989/90 y 2002/03 puede justificarse en base a que en esta última campaña comenzó a profundizarse la aplicación de SD, la cual cumple un rol fundamental en la disminución de la erosión.

Continuando con esta tendencia, se hubiese esperado que al seguir utilizando SD la pérdida de suelos entre 2002/03 y 2019/20 no se profundizara. Sin embargo, aunque no se presentan variaciones respecto al valor de A, el aumento de la pérdida de suelos en este último período puede entenderse como una consecuencia del incremento de la superficie destinada a los tres cultivos evaluados, el cual fue de alrededor del 16\%.

Por tanto, los resultados concuerdan con lo planteado por Oesterheld (2008), que afirma que el crecimiento de la pérdida de suelos por erosión es una de las principales consecuencias del aumento de la superficie agrícola; y lo dicho por López et al. (2015), quienes luego de evaluar una gran cantidad de lotes bajo $\mathrm{SD}$ en la región subhúmedasemiárida del sudoeste bonaerense, afirman que muchos de ellos presentan una cobertura menor a la necesaria para proporcionarle al suelo una correcta protección contra la erosión. 


\section{Análisis acerca de la pérdida económica}

Con referencia a la disminución real del rendimiento $(\mathrm{R})$, los valores conseguidos evidencian modificaciones según el escenario abordado y muestran concordancia con lo expresado por Pulido y Bocco (2011), quienes manifiestan que la erosión afecta directamente a la producción de cultivos. En este sentido, si tomamos lo ocurrido en el escenario I y lo comparamos con el II, podemos observar que, en las tres campañas abordadas, este último presenta aproximadamente un $50 \%$ menos de pérdida para los tres cultivos; como así también un detrimento económico inferior.

Dentro del escenario I, la pérdida de producción y su impacto económico registran valores opuestos entre ambos períodos. De esta manera, entre 1989/90 y 2002/03 se produce un decrecimiento del $27 \%$ de la merma productiva, repercutiendo en una disminución del $20 \%$ del perjuicio económico. En contraposición, entre 2002/03 y 2019/20 se observa una situación opuesta en ambos casos, manifestándose aumentos del 57\% de la pérdida de producción y del 149\% del impacto económico negativo.

De igual modo, en el escenario II, la disminución de la producción y su impacto económico también registran valores contrapuestos entre ambos períodos. Así, entre 1989/90 y 2002/03 se plantea una disminución del $23 \%$ de la caída productiva, repercutiendo en un descenso del 29\% del perjuicio económico; y, por el contrario, entre 2002/03 y 2019/20 se exhiben aumentos de la pérdida de producción y del impacto económico negativo, con valores similares a los planteado, en el mismo período del escenario I.

Tal como lo sucedido respecto de las modificaciones en los valores de A, la disminución en los valores de R entre 1989/90 y 2002/03-2019/20, podría deberse al reemplazo de la SC por SD, el cual fue más intenso a partir del año 2000, cumpliendo un rol de gran relevancia en el decrecimiento de la TME. Si bien en el segundo período esta práctica siguió siendo utilizada y la TME se mantuvo, la pérdida de producción creció, debido al ya mencionado incremento de la superficie cultivada.

Por consiguiente, aunque la SD permite disminuir la erosión (Sequeira, Vazquez \& Sacido, 2019), no es suficiente para revertir la situación observada, ya que la solución requiere más que un sencillo cambio del método de laboreo. Por ello, para lograr modelos agrícolas más sustentables, es necesario proyectar un rediseño de los agroecosistemas, donde además 
de considerar la SD, se concreten prácticas como el uso de cultivos de cobertura, la planificación de rotaciones, incorporación de materia orgánica, cultivar siguiendo las curvas de nivel e integrar la actividad ganadera, entre otras (Sicard \& Altieri, 2010).

Finalmente, con respecto a la pérdida económica, se destaca que los resultados obtenidos eran de esperarse, debido a la existencia de una relación directa entre la variación de la pérdida total de producción y la pérdida económica referida a la venta de los granos; la cual, a su vez, se ve profundizada por los constantes aumentos del dólar en el último período estudiado.

\section{Conclusiones}

El actual contexto marcado por la intensificación productiva, nos presenta un camino plagado de posibles impactos ambientales negativos. Teniendo en cuenta los efectos indeseados de este escenario, entre los que pueden destacarse aquellos generados en los procesos de erosión, se torna fundamental dimensionar el grado de afectación de los sistemas agroproductivos. En este sentido, la utilización de distintas clases de herramientas alcanza cada vez mayor importancia, siempre con el fin de enfrentar los desafíos en el abordaje de la obtención de sistemas productivos sustentables. Dentro de ellas, se encuentra la Ecuación Universal de Predicción de Erosión Hídrica, que ha demostrado ser un claro indicador para valorar la erosión en aquellas zonas destinadas a la agricultura.

En la presente investigación, el empleo de esta ecuación en el partido de Tres Arroyos en tres campañas agroproductivas, facilitó la estimación de la variación temporal de los valores de A y EP y de pérdida de suelos de las diversas series que componen las distintas UE definidas en el área. Asimismo, algunos de estos valores sirvieron como insumos para establecer un acercamiento a la pérdida económica ocasionada por el proceso de erosión.

En este contexto, en el cual el modelo agrícola hegemónico aumenta la vulnerabilidad de los agroecosistemas y los agricultores; el partido de Tres Arroyos manifiesta, desde la campaña 2002/03, un notable incremento en la pérdida total de producción, como consecuencia del crecimiento de la superficie sembrada y de la continuidad de procesos erosivos con características antrópicas, aún no solucionados. Sumado a ello, esto se traduce en un notable perjuicio económico, generando grandes pérdidas de 
ganancias; por lo que se torna apremiante desarrollar estrategias tendientes a revertir la mencionada situación y evitar que vuelva a producirse, estableciendo las bases para el inicio de una transición hacia un modelo de producción más sustentable.

Finalmente, se afirma que los resultados permiten detectar las áreas que se vieron más afectadas, tal es el caso de aquellas compuestas por las series TA y Lpd, y establecer cuáles deberían ser analizadas con mayor profundidad. Sumado a ello, se destaca que, al haber trabajado a escala de partido, se estaría teniendo en cuenta la totalidad de un área políticoadministrativa, lo cual resultaría sumamente significativo al momento de transmitir los resultados a aquellos tomadores de decisiones encargados de generar diversas medidas políticas, tendientes a implementar distintas medidas de mitigación.

\section{Referencias}

Armand, R., Bockstaller, C., Auzet, A. \& Van Dijk, P. (2009). Runoff generation related to intra-field soil surface characteristics variability. Application to conservation tillage context. En: Soil \& Tillage Research, 102, 27-37.

Bolsa de cereales. (2020). Cotización FOB actualizada de los principales cultivos argentinos. Recuperado de www.bolsadecereales.com.

Bouchoms, S., Wang, Z., Vanacker, V. \& Oost, K. V. (2019). Evaluating the effects of soil erosion and productivity decline on soil carbon dynamics using a model-based approach. Soil, 5(2), 367-382.

Carbone, M. E. \& Piccolo, M. C. (2002). Caracterización de las sequías en la localidad de tres arroyos (1930-1975). Aplicación del Método de Palmer. Papeles de geografía, (36), 209-222.

Casas, R. (2015). La erosión del suelo en la Argentina. En R. Casas y G. Albarracín (Eds.), Degradación de Tierras en la República Argentina. Buenos Aires: FECIC. (pp. 433-452).

Castilla, F. (2013). Siembra directa. La elegida para conservar el suelo. RIA. Revista de investigaciones agropecuaria, 39(2), 118-123.

Cerdá, E., Sarandón, S. \& Flores, C. (2014). El caso de "La Aurora": un ejemplo de aplicación del enfoque agroecológico en sistemas extensivos del sudeste de la provincia de Buenos Aires, Benito Juárez, Argentina. En S. Sarandón y C. Flores (Eds.), Agroecología: bases 
teóricas para el diseño y manejo de Agroecosistemas sustentables (437-463). La Plata: Servicio de publicaciones, UNLP.

Colombo, S. (2004). Valoración y análisis económico de impactos ambientales en procesos erosivos: aplicación de los métodos de valoración contingente y experimento de elección en la cuenca del Alto Genil. Cuadernos Geográficos, (34), 223-232.

Concepción Ramos, M., Lizaga, I., Gaspar, L., Quijano, L. \& Navas, A. (2018). Soil and nutrient losses by hydric erosion under different land uses in a mountain catchment in the Ebro Basin (NE Spain). EGU General Assembly Conference Abstracts. 4 al 13 de abril. Viena, Austria.

Cotler, H., López, C. A., \& Martínez-Trinidad, S. (2011). ¿Cuánto nos cuesta la erosión de suelos? Aproximación a una valoración económica de la pérdida de suelos agrícolas en México. Investigación ambiental, 3(2), 31-43.

Cristeche, E. (2009). Valoración económica de los efectos externos de la erosión hídrica sobre la infraestructura de caminos rurales en el Sur de la provincia de Córdoba, Argentina. (Tesis de Maestría en economía). Facultad de Cs. Económicas, Universidad de Buenos Aires, Argentina.

DAA (Datos Abiertos Agroindustria). (2020). Estadísticas agrícolas suministradas por la Secretaría de Agroindustria, Ministerio de Producción y Trabajo, República Argentina. Recuperado de https://datos. agroindustria.gob.ar.

Díaz Pereira, E., Anaya Romero, M. \& de la Rosa Villafranca, D. (2011). Modelos de evaluación agroecológica de tierras: erosión y contaminación en el entorno MicroLEIS. Teoría y Praxis, (9), 91-107.

Food and Agriculture Organization of the United Nations. (FAO). (1990). Guidelines for soil description. Roma, Italia: FAO.

Fundación para la Educación, la Ciencia y la Cultura (FECIC)-Centro para la Promoción de la Conservación del Suelo y del Agua (PROSA). (1988). El Deterioro del Ambiente en la Argentina. Buenos Aires, Argentina: Editorial Orientación Grafica Editora.

Ferreras, L. A., Toresani, S. M. I., Faggioli, V. S. \& Galarza, C. M. (2015). Sensibilidad de indicadores biológicos edáficos en un Argiudol de la Región Pampeana Argentina. Sociedad Española de la Ciencia del Suelo, 5 (3), 227-242. 
Gaitán, J., Navarro, M. F., Vuegen, L. T., Pizarro, M. J., Carfagno, P. \& Rigo, S. (2017). Estimación de la pérdida de suelo por erosión hídrica en la República Argentina. Buenos Aires, Argentina: INTA Ediciones.

García Ruiz, V., Savé, R. \& Herrera, A. (2013). Análisis multitemporal del cambio de uso del suelo, en el Paisaje Terrestre Protegido Miraflor Moropotente Nicaragua, 1993-2011. Ecosistemas, 22(3), 117-126.

Gvozdenovich, J., Barbagelata, P. \& López, G. (2015). Erosión Hídrica USLE/RUSLE INTA EEA Paraná, Argentina. Software, Versión 2.0. Recuperado de http://www.inta.gob.ar/parana

Gvozdenovich, J., Pérez Bidegain, M., Novelli, L., \& Barbagelata, P. (2017). ¿Puede WEPP mejorar la predicción de la erosión de suelos respecto a USLE? Ciencia del suelo, 35(2), 259-272.

Hernández, L., \& Dufilho, A. C. (2012). Determinación del riesgo de erosión hídrica de los suelos de los departamentos Minas y Chos Malal (Neuquén) mediante la aplicación de la ecuación universal de pérdida de suelo (USLE). Boletín geográfico, (34), 11-31.

Instituto Nacional de Tecnología Agropecuaria (INTA). (1990). Atlas de suelos de la república argentina. E: 1:500.000 y 1:1000.000. Buenos Aires, Argentina: Secretaría de Agricultura, Ganadería y Pesca, Proyecto PNUD ARG 85/019.

Irurtia, C. \& Cruzate, G. (2002). Aplicación de la Ecuación Universal de Pérdida de Suelo en la Provincia de Buenos Aires. Informe Técnico. Castelar, Buenos Aires: INTA.

Leal Villamil, J. \& Lozano Botache, L. A. (2012). Niveles de fragilidad potencial para la erosión y el deslizamiento en los suelos del municipio de Ibagué (Tolima). Revista de Investigación Agraria y Ambiental, 3(1), 67-76.

López, F. M., Duval, M. E., Martínez, J. M. \& Galantini, J. A. (2015). Cobertura en el sudoeste bonaerense en suelos bajo siembra directa. Ciencia del suelo, 33(2), 273-281.

Mikkelsen, C. A. (2013). Debatiendo lo rural y la ruralidad: un aporte desde el sudeste de la provincia de Buenos Aires; el caso del partido de Tres Arroyos. En: Cuadernos de Geografía-Revista Colombiana de Geografía, 22(2), 235-256.

Nájera, O., Bojórquez, J. I., Flores, F., Murray, R. M. \& González, A. (2016). Riesgo de erosión hídrica y estimación de pérdida de suelo 
en paisajes geomorfológicos volcánicos en México. Cultivos Tropicales, 37(2), 45-55.

Nearing, M. A., Xie, Y., Liu, B. \& Ye, Y. (2017). Natural and anthropogenic rates of soil erosion. In: International Soil and Water Conservation Research, 5(2), 77-84.

Oesterheld, M. (2008). Impacto de la agricultura sobre los ecosistemas: fundamentos ecológicos y problemas más relevantes. Ecología austral, 18(3), 337-346.

Pulido J. \& Bocco, G. (2011). ¿Cómo se evalúa la degradación de tierras? Panorama global y local. Interciencia, 36 (2), 96-103.

Sartori, M., Philippidis, G., Ferrari, E., Borrelli, P., Lugato, E., Montanare1la, L. \& Panagos, P. (2019). A linkage between the biophysical and the economic: Assessing the global market impacts of soil erosion. Land use policy, 86, 299-312.

Sicard, T. E. \& Altieri, M. A. (2010). Vertientes del pensamiento agroecológico: Fundamentos y Aplicaciones. Bogotá, Colombia: Opciones Gráficas Editores.

Sequeira, N. D., Vazquez, P. \& Sacido, M. (2019). Erosión hídrica y su relación con el avance de la agricultura en el sudeste bonaerense, Argentina. El caso del partido de Benito Juárez durante las campañas 1989/90, 2002/03 y 2014/15. Papeles de Geografía, (65), 106-120.

Toy, T. J., Foster, G. R. \& Renard, K. G. (2002). Soil erosion: processes, prediction, measurement, and control. Nueva York, USA: John Wiley and Sons.

Vázquez, P. (2004). Comparación temporal de la sustentabilidad de dos modalidades de producción agrícolas (Tandil, Argentina). Tesis de Licenciatura en Diagnóstico y Gestión Ambiental, Facultad de Cs. Humanas, Universidad Nacional del Centro de la Provincia de Buenos Aires, Argentina.

Vázquez, P., Zulaica, L., Mickelsen, C. \& Sacido, M. (2018). Zonificación ecológica y expansión agropecuaria en el partido de Tres Arroyos: un estudio de caso en la Región Pampeana Argentina. V Jornadas Nacionales de Investigación en Geografía Argentina y XI Jornadas de Investigación y Extensión del Centro de Investigaciones Geográficas. 16 al 19 de mayo. Facultad de Cs. Humanas, Universidad Nacional del Centro de la Provincia de Buenos Aires, Argentina. 
Vazquez, P., Ruiz, D., Zulaica, L., Sequeira, N. D. \& Daga, D. Y. (2019). Paisajes agroproductivos y sustentabilidad agrícola en un Partido de la Región Pampeana Austral: análisis de las transformaciones del territorio rural entre 2002 y 2015. En: I Congreso Argentino de Agroecología. 18 al 20 de septiembre. Facultad de Cs. Agrarias, Universidad Nacional de Cuyo. Mendoza, Argentina.

Villanueva, A., Cazenave, G. y Bilello, G. (2009). Valor económico del control de inundaciones en el Partido de Tres Arroyos, Pcia. de Buenos Aires, Argentina. REGA, 6(1), 43-53.

Wischmeier, W. H. \& Smith, D. D. (1978). Predicting rainfall erosion losses. A guide to conservation planning. Maryland, USA: Science and Education Administration. 